\title{
Self-Assembled Nanomaterials Based on Perfluorophenyl-Capped Dipeptides
}

\author{
Yu-Chun Lin, Shu-Min Hsu, Jui-Wen Chang, Yu-Hao Liu, and Hsin-Chieh Lin
}

\begin{abstract}
Herein, we report a series of low-molecular-weight hydrogelators and prove that the phenyl-perfluorophenyl pair in the structure of the hydrogelators can be used to promote the formation of the supramolecular hydrogels in physiological condition. Further spectroscopic study indicates the aromatic-aromatic and hydrogen-bonding interactions might be the major driving force behind the self-assembly of the nanostructured hydrogels. Biocompatible experiments of the hydrogelators were carried out on HeLa and MCF-7 cells which indicate the newly discovered hydrogelators are potential biomaterials.
\end{abstract}

Index Terms-Phenyl-perfluorophenyl pair, hydrogel, nanofiber, biomaterials.

\section{INTRODUCTION}

Self-assembly of proteins occurs in nature and therefore peptide hydrogels are believed to be one of the most useful strategies for developing new biomaterials. [1] The potential of peptide hydrogels are huge because these hydrogels have a defined but tunable composition and architecture. [2] Now, a large number of application based on peptide hydrogels have been explored, including scaffolds for tissue engineering, [3]-[5] vehicles for drug and gene delivery, [6]-[8] matrices for enzyme assay, [9] and topical wound treatments and pain relievers. [10] The formation of a supramolecular hydrogel is a balance between crystallization and water solubilization. The basic design of this type of materials are the use of noncovalent intermolecular interactions, including $\pi-\pi$ stacking, hydrogen bonding, electrostatic interactions, van der Waals forces and other relatively weak interactions, to facilitate the formation of supramolecular hydrogels via self-assembly.

There are three major classes of peptide hydrogels, including low molecular weight (small molecule) hydrogels, [11]-[18] ionic complimentary peptides [19], [20] and peptide amphiphiles. Among these, the low molecular weight hydrogels are relative useful if one aims to elucidate the structure-property relationship. Nowadays, one of the major

Manuscript received May 10, 2014; revised July 12, 2014. This work has been financially supported by the National Science Council of the Republic of China, Taiwan (Grant No. NSC 102-2113-M-009-006-MY2). This work is also supported by the "Aim for the Top University" program of the National Chiao Tung University and Ministry of Education, Taiwan, R.O.C.. We are grateful to the National Center for High-performance Computing of Taiwan for computer time and facilities. Additionally, we thank Prof. Shiaw-Guang Hu for technical support

Yu-Chun Lin, Shu-Min Hsu, Jui-Wen Chang, Yu-Hao Liu and Hsin-Chieh Lin are with the Department of Materials Science and Engineering, National Chiao Tung University, Taiwan (e-mail: hclin45@ nctu.edu.tw). strategies for constructing a low molecular weight hydrogelator is to combine a short peptide structure and an end-capped $\pi$-conjugated system such as fluorenylmethoxycarbonyl (Fmoc) or naphthyl (Nap) [21].

The supramolecular synthon, perfluoroarene-arene pairs, have recently attracted considerable interest because this motif is widely used for designing structures of crystals and molecular assemblies. [22]-[27] It has been shown that the perfluoroarene-arene pairs are useful supramolecular synthon in crystal engineering and reaction control. In our previous work, [28] we have shown that the first example of intramolecular binding of phenyl-perfluorophenyl pair in the peptide structures for the formation of supramolecular hydrogels. As previously demonstrated, the presence of the sandwiched water between aromatic rings may assist the formation of intramolecular phenyl-perfluorophenyl complex which facilitates the formation of supramolecular hydrogels. In this work, a detail discussion of the self-assembly nanostructures, mechanical properties, cell viabilities and wound-healing abilities for perfluorophenyl-capped dipeptides (PFB-peptides) are presented.

\section{MATERIALS AND METHODS}

\section{A. Synthesis}

The hydrogelators 1-3 were synthesized according to previous report. [28] The dipeptide derivative 1 was prepared by solid phase peptide synthesis (SPPS) using 2-chlorotrityl chloride resin (100-200 mesh and 0.3-0.8 mmol/g), Fmoc-glycine, Fmoc- ${ }_{-}-$phenylalanine (Fmoc- $\left.{ }_{-}-\mathrm{Phe}\right)$ and Pentafluoro benzeneacetic acid. The dipeptide derivatives 2 and 3 were prepared by replacing Fmoc-glycine with Fmoc- ${ }_{-}$-alanine and $\mathrm{Fmoc}_{-}-$-valine, respectively.

\section{B. Inverted Tube Method}

The gelation experiment was performed by carefully weighing $1.0 \mathrm{mg}$ of compound in a $2 \mathrm{~mL}$ vial (diameter 10 $\mathrm{mm}$ ) with a screw cap and the addition of $0.10 \mathrm{~mL}$ of the solvent. The vial was sealed tightly and heated until the compound dissolved, at which time the vial was cooled to room temperature. Gelation is considered to have occurred when a solid-like material is obtained that does not exhibit gravitational flow (inverted test tube method). The determination of gelation was made by tilting the bottles for 5 min. If there was no flow, the sample was considered being gelled.

\section{Rheological Tests}

Rheological tests were carried out on Anton Paar rheometer. A $25 \mathrm{~mm}$ parallel plate was used for the 
experiment. $200 \mu \mathrm{L}$ of hydrogel sample (1 wt \%) was placed on the parallel plate. Angular frequency sweep test: Test range $\left(0.1\right.$ to $100 \mathrm{rads}^{-1}$ frequency, strain $\left.=0.8 \%\right), 13$ points per decade. Sweep mode is "log" and temperature was carried out at $25^{\circ} \mathrm{C}$.

\section{Cell Viability Tests}

The biocompatibilities of different peptides were measured by the MTT cell proliferation assay. The HeLa/MCF-7 cells were seeded in 24-well plates at a density of 50000 cells per well with $0.5 \mathrm{~mL}$ DMEM contained $10 \%$ FBS and $1 \%$ Penicillin and incubated for overnight. Compounds at different concentrations $(10,50,100,200,500 \mu \mathrm{M})$ were added when cells were plated. 24 and $48 \mathrm{~h}$ later, replaced the medium with $0.5 \mathrm{~mL}$ per well of fresh medium contained 10 $\%$ MTT reagent $\left(5 \mathrm{mg} \mathrm{mL}^{-1}\right)$. After $4 \mathrm{~h}$ of incubation at $37{ }^{\circ} \mathrm{C}$ in a $5 \% \mathrm{CO}_{2}$ incubator, the medium containing MTT was removed and DMSO was added to dissolve the formazan crystals and aliquots were pipetted into a 96-well plate. The optical density of the result solution was measured at $595 \mathrm{~nm}$, using an absorbance microplate reader (Infinite F50, TECAN). Cells without the treatment of the compounds were used as the control. The cell viability percentage was calculated by the following formula: The cell viability percentage $(\%)=\mathrm{OD}_{\text {sample }} / \mathrm{OD}_{\text {control }} \times 100 \%$.

\section{E. Wound-Healing Tests}

HeLa/MCF-7 cells were re-suspended in T-75 tissue culture flask after washing cells twice with PBS. The cells were detached by $5 \mathrm{~mL} \quad 0.25 \%$ trypsin containing $0.1 \%$ EDTA and the cells were re-suspended with $5 \mathrm{~mL}$ complete medium. After centrifuging for $3 \mathrm{~min}$, the viable cells were counted by trypan blue assay. 30000 cells (in $3 \mathrm{~mL}$ medium) were plated into each vial on a 6-well plate to create a confluent monolayer. After adherent for $24 \mathrm{~h}$, a wound was created by scraping the cell monolayer with a p200 pipet tip. The cells were washed twice with $2 \mathrm{~mL}$ of PBS to remove flowing cells and replace with $3 \mathrm{~mL}$ complete medium. The 0 $\mathrm{h}$ image was acquired as a reference point. The medium was replaced with $3 \mathrm{~mL}$ of medium containing $1 \mathrm{wt} \%$ of hydrogelators and the plate was incubated at $37{ }^{\circ} \mathrm{C}, 5 \% \mathrm{CO}_{2}$ for $24 \mathrm{~h}$ and $48 \mathrm{~h} .0 \mathrm{~h}, 24 \mathrm{~h}$ and $48 \mathrm{~h}$ images were acquired at the match photographed region.

\section{RESULTS AND DISCUSSION}

\section{A. Chemical and Physical Properties of Hydrogels}<smiles>[R2]C(NC(=O)C([R2])NC(=O)Cc1c(F)c(F)c(F)c(F)c1F)C(=O)O</smiles>

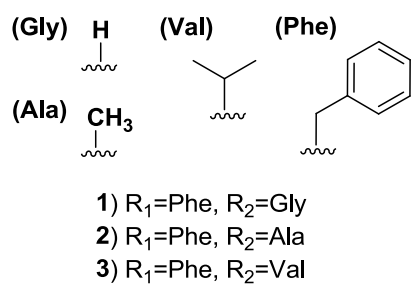

Fig. 1. Chemical structures of peptide-based hydrogelators 1-3.
The dipeptides 1-3 were synthesized according to the solid-phase peptide synthesis (SPPS) and their chemical structures were shown in Fig. 1. The formation of the hydrogels of 1-3 was carried out by tuning the $\mathrm{pH}$ values and the resulting optical images are collected in insets of Fig. 2. The appearances of the hydrogels are transparent gel for 1 and 2 , and semi-translucent gel for 3. The microscopic nanostructures of the negative stained hydrogels were measured by transmission electron microscopy (TEM) and collected in Fig. 2. Dissolving the dipeptide 1 at a concentration of $1 \mathrm{wt} \%$ and $\mathrm{pH} 7.0$ in aqueous solution led to the formation of the hydrogel, thus indicating self-assembly and hydrogelation occurred in 1 . The dipeptides of 2 and 3 formed supramolecular hydrogels at the concentration of 1 wt $\%$ at $\mathrm{pH} 6.0$, showing the generality of the use of the $\mathrm{PFB}_{-}-\mathrm{Phe}$ in the structural design of the peptide-based hydrogelators. The hydrogelation tests of the dipeptides 1-3 were found to be dependent on $\mathrm{pH}$ values (Table I), which can be explained by the equilibrium between carboxylic acid and carboxylate. The TEM analysis revealed that the hydrogels 1-3 consisted of a fibrous network with fibril diameters of $8 \sim 14 \mathrm{~nm}$ (Fig. 3) and these nanofibers entangle to trap water and result in hydrogels. These observations indicate self-assembly and hydrogelation occurred in 1-3.

TABLE I: PHySICAL PROPERTIES OF PEPTIDE-BASEd HydROGELATORS

\begin{tabular}{cclcc}
\hline \hline Entry & \multirow{2}{*}{ Ap } & Appearance & $\begin{array}{c}\text { G', G’ } \\
(\mathrm{Pa})\end{array}$ & $\begin{array}{c}\text { Fiber width } \\
(\mathrm{nm})\end{array}$ \\
\hline \multirow{2}{*}{$\mathbf{1}$} & \multirow{2}{*}{7.0} & \multirow{2}{*}{ Transparent gel } & $\begin{array}{c}1.2 \times 10^{4}, \\
1.5 \times 10^{3}\end{array}$ & $8 \pm 1$ \\
& & & $4.4 \times 10^{3}$, & \\
\multirow{2}{*}{$\mathbf{2}$} & \multirow{2}{*}{6.0} & Transparent gel & $3.0 \times 10^{2}$ & $10 \pm 2$ \\
& & & $3.2 \times 10^{3}$, & \\
\multirow{3}{*}{6} & \multirow{2}{*}{6.0} & Semi-translucent gel & $8.7 \times 10^{1}$ & $14 \pm 2$ \\
\hline \hline
\end{tabular}

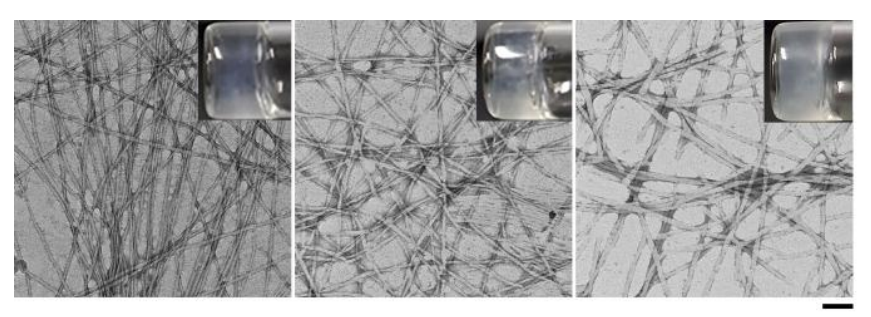

a)

b)

c)

Fig. 2. Optical and negative stained TEM images of hydrogels for a) 1, b) 2 , and c) 3 at the concentration of $1 \mathrm{wt} \%($ scale bar $=100 \mathrm{~nm})$.

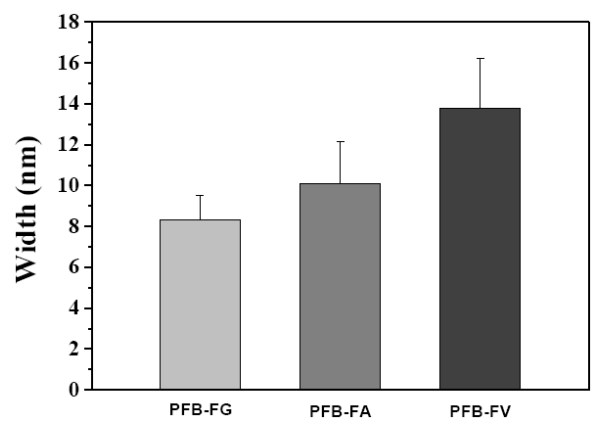

Fig. 3. The average fiber diameters of 1-3.

The UV-vis absorption and circular dichroism (CD) spectra were measured to explore the superstructures of these self-assembled hydrogels. As shown in Fig. 4a-c, the low-energy UV-vis absorption profiles of hydrogels 1-3 revealed at 260-330 $\mathrm{nm}$, which are consistent with the 
aromatic $\pi-\pi^{*}$ transitions. In Fig. $4 \mathrm{a}$ and $4 \mathrm{~b}$, both the CD spectra of the hydrogels of 1 and 2 showed bisignated Cotton effects, with a negative part at $261 \mathrm{~nm}$ and a positive part at $287 \mathrm{~nm}$, thus suggesting the interacting $\pi$-conjugated systems in an ordered arrangement. While with increasing the bulkiness of the aliphatic side chain of the amino acid, such as 3, there are fewer modes of aromatic-aromatic interactions (260-330 nm) between phenyl and pentafluorophenyl rings (Fig. 4c). Further detail analysis of the CD spectrum is also an useful tool to study the formation of the extended hydrogen-bonding interactions such as $\beta$-sheet or -turn structure. The CD spectra of dipeptide hydrogels of 1-3 revealed few negative peaks in the range of 215-240 nm, thus implying the possibility of the formation of $\beta$-sheet and/or $\beta$-turn structures. Fourier transform infrared (FT-IR) was used for the analysis of the secondary structures of the self-assembly nanostructures in supramolecular hydrogels. As shown in Fig. 5a, FT-IR spectrum of the hydrogels of 1, 2 and 3 gels showed two peaks located around $1656 \mathrm{~cm}^{-1}, 1630 \mathrm{~cm}^{-1}$ and a less intense peak around $1545 \mathrm{~cm}^{-1}$, which is the characteristics of extended $\beta$-sheet structures. The fluorescence emission spectra showed that there appears to be a red-shift in the emission $(\sim 350 \mathrm{~nm})$ in the gel state, thus indicating $\pi-\pi$ interactions between aromatic rings occurred in the hydrogels of 1-3. [28] Based on the spectroscopic characterizations of dipeptide hydrogels of 1-3, the cooperative effect of extended aromatic-aromatic and extended hydrogen-bonding interactions might constitute the major driving force in the formation of the self-assembly supramolecular hydrogels.
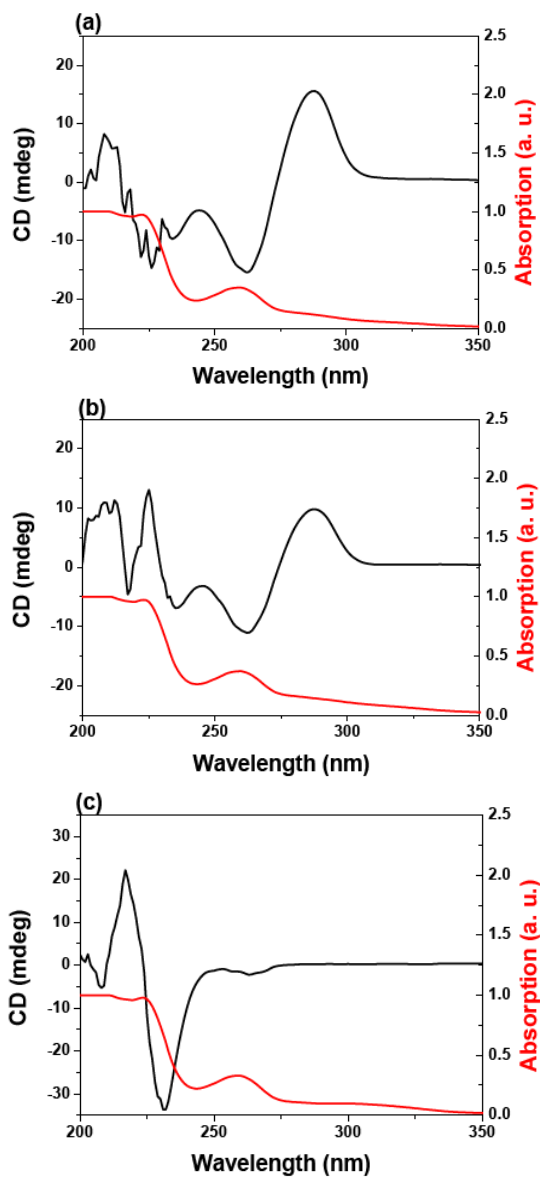

Fig. 4. CD (black line) and UV-vis absorption (red line) spectra of (a) 1, (b) 2 , and (c) 3 at $0.1 \mathrm{wt} \%$ solution.
It is believed that the supramolecular hydrogels are potentially useful biomaterials for use in the the application of tissue engineering. [6] In terms of the hydrogels as 3D environment for cell growth, the following criteria should be reached: (i) the $\mathrm{T}_{\text {gel-sol }}$ (gel-to-sol transition temperature) of the hydrogel should be higher than the physiologically relevant temperature (i.e., $37{ }^{\circ} \mathrm{C}$, body temperature), (ii) the mechanical property of the hydrogel can support the mass of cells, (iii) the hydrogel materials should be biocompatible. In general, because live cells are usually cultured at $37{ }^{\circ} \mathrm{C}$, the $\mathrm{T}_{\text {gel-sol }}$ of a $3 \mathrm{D}$ culture medium needs to be higher than this temperature. All gel-to-sol transition temperatures of 1, 2 and 3 are $48{ }^{\circ} \mathrm{C}, 48{ }^{\circ} \mathrm{C}$, and $46{ }^{\circ} \mathrm{C}$, respectively, pointing to potential uses of these hydrogels as scaffolds for tissue engineering. The mechanical property of a hydrogel can be evaluated by oscillatory rheology. It provides two essential parameters for hydrogels; they are the elastic and the viscous responses which can be denoted as storage modulus $\left(\mathrm{G}^{\prime}\right)$ and loss modulus $\left(\mathrm{G}^{\prime \prime}\right)$, respectively. Materials with storage moduli of around $100 \mathrm{~Pa}$ are thought to be compatible with culture of soft tissue cells such as neurons and certain epithelial cells. As shown in Fig. 5 and Table I, the storage moduli of hydrogels 1,2 , and 3 are found to be higher than their loss moduli, indicating that these three materials are elastic. The elastic storage moduli observed for 1, 2, and 3 were $12 \mathrm{KPa}, 4.4 \mathrm{KPa}$, and $3.2 \mathrm{KPa}$, respectively. These results indicate that the hydrogels are sufficient to support the mass of a cell. The storage moduli of dipeptides 1 exhibits the highest storage modulus among these hydrogels, thus implying the relative thinner nanofibers $(8 \mathrm{~nm})$ may cause more significant physical cross-links and entanglements in the system.

\section{(a)}

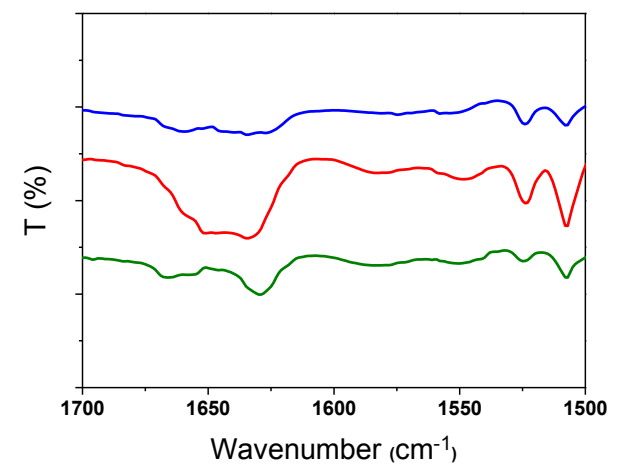

(b)

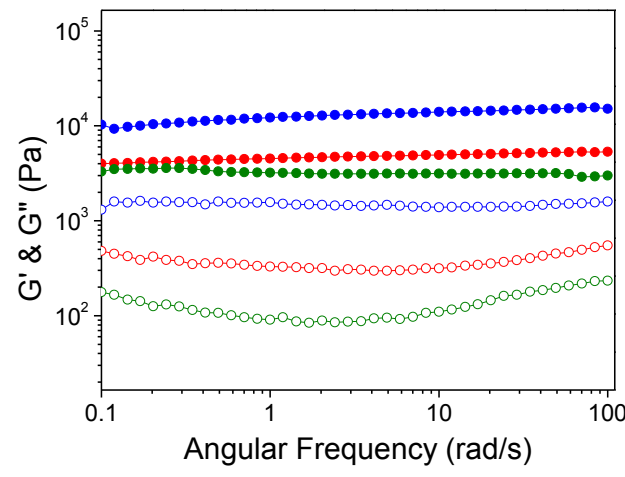

Fig. 5. (a) FT-IR spectra of the hydrogels of 1 (blue), 2 (red), and 3 (green). (b) The frequency sweep of the hydrogels of 1 (blue), 2 (red), and 3 (green). All the concentration of hydrogels are prepared at $2 \mathrm{wt} \%$; closed circles for G' and open circles for G'. 


\section{B. Biology Study}

Supramolecular hydrogels of peptides 1-3 were subsequently tested for their ability to support the biocompatibilities of HeLa and MCF-7 cells. The viability tests of HeLa cells exposed to different concentrations of 1-3 $(10$ to $500 \mu \mathrm{M})$ for $48 \mathrm{~h}$ were estimated by MTT dye (3-(4,5-dimethylthiazol-2-yl)-2,5-diphenyltetrazolium bromide) assay. As shown in Fig. 6, the experiments revealed that after being incubated with the hydrogelators 1,2 , and 3 for $48 \mathrm{~h}, \mathrm{HeLa}$ cells that were grown in liquid medium showed the proliferation capacities. The concentrations of hydrogels 1 , 2 , and 3 required for $50 \%$ inhibition $\left(\mathrm{IC}_{50}\right)$ are higher than $500 \mu \mathrm{M}$, thus indicating these hydrogelators are biocompatible. [13] In addition, the survival ratio of 1 was above $80 \%$ at $500 \mu \mathrm{M}$, and its morphologies are similar to the control cells that were grown in the medium without hydrogelators. Furthermore, we used a wound-healing assay to examine the capability of the hydrogels of 1 where the cell/matrix interactions are held. The transparent hydrogels have the advantage in that the wound-healing assay can be easily monitored without removing the dressing. As shown in Fig. 7, the presence of the hydrogelor of 1 in the cell culture show the similar results on the migration of the HeLa cells as compared with that of the reference (Fig. 7b), which further supports the biocompatibility of 1 . Since the compound 1 showed the relative biocompatible ability among 1-3, we further study the cytotoxicity of 1 (10 to $500 \mu \mathrm{M})$ in MCF-7 cells for 48 h. Fig. 8 showed the viability of MCF- 7 cells after $48 \mathrm{~h}$ post treatment and showed good viability which is consistent with $500 \mu \mathrm{M}$ of 1 in HeLa cells. Fig. 9 showed the results of the wound-healing test of 1 with the MCF-7 cells at 0,24 and $48 \mathrm{~h}$. The presence of the hydrogelors of 1 in the cell culture showed little inhibitory effect $\left(\mathrm{L}_{24 \mathrm{~h}} / \mathrm{L}_{0 \mathrm{~h}}, \mathrm{~L}\right.$ : wound length) on the migration of the MCF-7 cells as compared with that of the reference (Fig. 9b) at 24h. After $48 \mathrm{~h}$, the morphologies in MCF-7 and HeLa cells were similar to that of reference. These results suggest the compound 1 might be a relatively potential biomaterial in comparison with 2 and 3 .
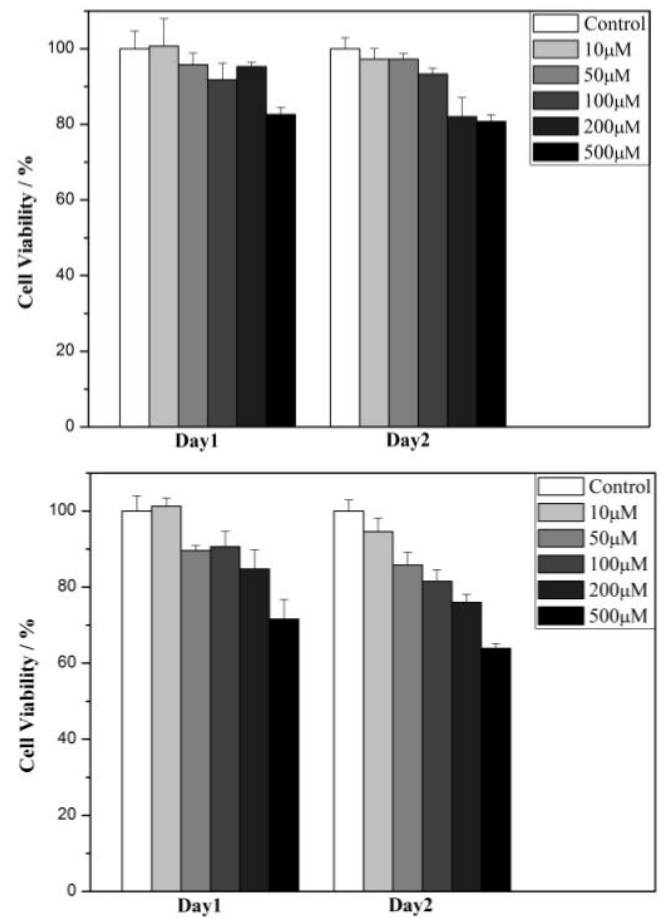

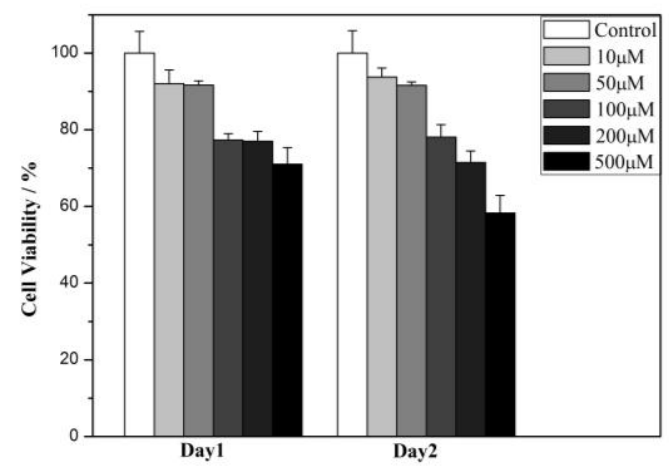

Fig. 6. Viability of HeLa cells incubated with $10,50,100,200$, and $500 \mu \mathrm{M}$ of 1 (upper), 2 (middle), and 3 (lower) after 24 and $48 \mathrm{~h}$. (a)
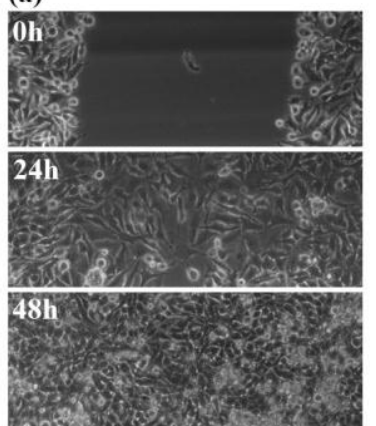

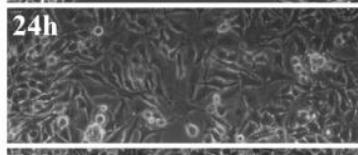

(b)

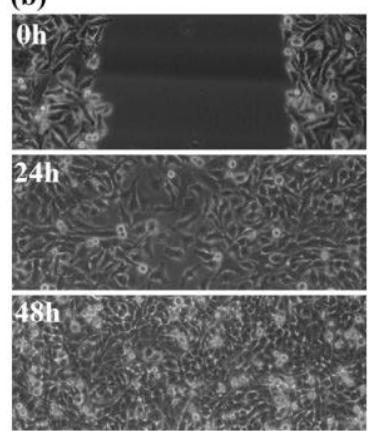

Fig. 7. Optical images of HeLa cells on the surface $0 \mathrm{~h}$ (upper), $24 \mathrm{~h}$ (middle) and $48 \mathrm{~h}$ (lower) after creation of scratches in the presence of $1 \mathrm{wt} \%$ of hydrogels (a) 1 and (b) reference.

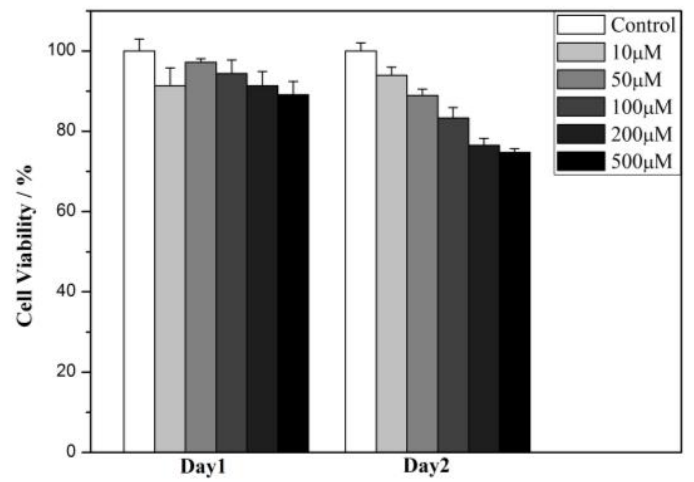

Fig. 8. Viability of MCF-7 cells incubated with 10, 50, 100, 200, and 500 $\mu \mathrm{M}$ of 1 after 24 and $48 \mathrm{~h}$. (a)

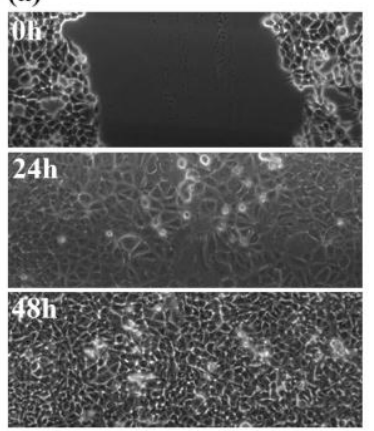

(b)

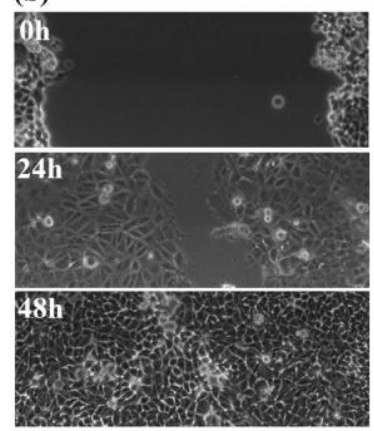

Fig. 9. Optical images of MCF-7 cells on the surface $0 \mathrm{~h}$ (upper), $24 \mathrm{~h}$ (middle) and $48 \mathrm{~h}$ (lower) after creation of scratches in the presence of 1 wt $\%$ of hydrogels a) 1 and b) reference.

\section{CONCLUSION}

In summary, we have developed a series of low-molecular-weight hydrogelators and proved that the phenyl-perfluorophenyl pair can be used to promote the 
formation of the supramolecular hydrogels. The self-assembly nanostructures in hydrogels were confirmed by transmission electron microscopy (TEM), circular dichroism (CD), Fourier transform infrared (FT-IR), UV-vis absorption as well as fluorescence spectroscopy. Based on the spectroscopic data, the formation of extended self-assembled nanostructures occurs at the gelation condition for dipeptide hydrogelators, indicating the aromatic-aromatic and hydrogen-bonding interactions may be the major driving force behind the self-assembly of the nanofibers and hydrogels. The structural and physical properties of peptide hydrogels were found to be dictated by the peptide sequence. These results indicate that HeLa and MCF-7 cells have good biocompatibilities with PFB-capped hydrogels especially for 1. Based on our study, the newly discovered hydrogel of $\mathbf{1}$ is potential biomaterials. This work illustrates the importance of structure-property relationship for their further applications in in vivo biomedical applications.

\section{APPENDIX}

Appendixes, if needed, appear before the acknowledgment.

\section{ACKNOWLEDGMENT}

We would like to thank Prof. Shiaw-Guang $\mathrm{Hu}$ for technical support. This work has been financially supported by the National Science Council of the Republic of China, Taiwan (Grant No. NSC 102-2113-M-009-006-MY2). This work is also supported by the "Aim for the Top University" program of the National Chiao Tung University and Ministry of Education, Taiwan, R.O.C.. We are also grateful to the National Center for High-performance Computing of Taiwan for computer time and facilities.

\section{REFERENCES}

[1] H.-C. Lin and B. Xu, Applications of Supramolecular Chemistry, CRC, Boca Raton, FL, 2012, ch. 13.

[2] J. B. Matson and S. I. Stupp, "Self-assembling peptide scaffolds for regenerative medicine," Chem. Commun., vol. 48, pp. 26-33, Nov. 2012.

[3] A. Mahler, M. Reches, M. Rechter, S. Cohen, and E. Gazit, "Rigid, self-assembled hydrogel composed of a modified aromatic dipeptide," Adv. Mater., vol. 18, pp. 1365-1370, Jun. 2006.

[4] K. M. Galler, L. Aulisa, K. R. Regan, R. N. D'Souza, and J. D. Hartgerink, "Self-Assembling multidomain peptide hydrogels: designed susceptibility to enzymatic cleavage allows enhanced cell migration and spreading," J. Am. Chem. Soc., vol. 132, pp. 3217-3223, Feb. 2010.

[5] A. M. Smith, R. J. Williams, C. Tang, P. Coppo, R. F. Collins, M. L. Turner, A. Saiani, and R. V. Ulijn, "Fmoc-diphenylalanine self assembles to a hydrogel via a novel architecture based on $\pi-\pi$ interlocked $\beta$-sheets," Adv. Mater., vol. 20, pp. 37-41, Jan. 2008.

[6] H. Wang, J. Wei, C. Yang, H. Zhao, D. Li, Z. Yin, and Z. Yang, "The inhibition of tumor growth and metastasis by self-assembled nanofibers of taxol," Biomaterials, vol. 33, pp. 5848-5853, Aug. 2012.

[7] X. Li, Y. Kuang, J. Shi, Y. Gao, H.-C. Lin, and B. Xu, "Multifunctional, biocompatible supramolecular hydrogelators consist only of nucleobase, amino acid, and glycoside," J. Am. Chem. Soc., vol. 133, pp. 17513-17518, Sep. 2011.

[8] H. Komatsu, S. Matsumoto, S. Tamaru, K. Kaneko, M. Ikeda, and I. Hamachi, "Supramolecular hydrogel exhibiting four basic logic gate functions to fine-tune substance release," J. Am. Chem. Soc., vol. 131, pp. 5580-5585, Mar. 2009.
[9] Z. Yang, K. Xu, Z. Guo, Z. Guo, and B. Xu, "Intracellular enzymatic formation of nanofibers results in hydrogelation and regulated cell death," Adv. Mater., vol. 19, pp. 3152-3156, Oct. 2007.

[10] Z. Yang, G. Liang, M. Ma, A. S. Abbah, W. W. Lu, and B. Xu, "D-Glucosamine-based supramolecular hydrogels to improve wound healing," Chem. Commun., vol. 843, pp. 843-845, Dec. 2007.

[11] K. M. Galler, L. Aulisa, K. R. Regan, R. N. D’Souza, and J. D. Hartgerink, "Self-assembling multidomain peptide hydrogels: designed susceptibility to enzymatic cleavage allows enhanced cell migration and spreading," J. Am. Chem. Soc., vol. 132, pp. 3217-3223, Feb. 2010.

[12] J. Li, Y. Kuang, Y. Gao, X. Du, J. Shi, and B. Xu, "D-amino acids boost the selectivity and confer supramolecular hydrogels of a nonsteroidal anti-inflammatory drug (NSAID)," J. Am. Chem. Soc. Vol. 135, pp. 542-545, Nov. 2013.

[13] F. Zhao, M. L. Ma, and B. Xu, "Molecular hydrogels of therapeutic agents," Chem. Soc. Rev., vol. 38, pp. 883-891, Feb. 2009.

[14] Z. Yang, G. Liang, and B. Xu, "Enzymatic hydrogelation of small molecules," Acc. Chem. Res., vol. 41, pp. 315-326, Jan. 2008.

[15] Y. Gao, M. J. C. Long, J. F. Shi, L. Hedstrom, and B. Xu, "Using supramolecular hydrogels to discover the interactions between proteins and molecular nanofibers of small molecules," Chem Commun., vol. 48, pp. 8404-8406, Jul. 2012.

[16] A. Wada, S. Tamaru, M. Ikeda, and I. Hamachi, "MCM-enzyme-supramolecular hydrogel hybrid as a fluorescence sensing material for polyanions of biological significance," J. Am. Chem. Soc., vol. 131, pp. 5321-5330, Mar. 2009.

[17] S. C. Bremmer, J. Chen, A. J. McNeil, and M. B. Soellner, "A general method for detecting protease activity via gelation and its application to artificial clotting," Chem Commun., vol. 48, pp. 5482-5484, Mar. 2012.

[18] X. Li, Y. Kuang, H.-C. Lin, Y. Gao, J. Shi, and B. Xu, "Supramolecular nanofibers and hydrogels of nucleopeptides," Angew. Chem. Int. Ed., vol. 50, pp. 9365-9369, Sep. 2011.

[19] S. Zhang, "Lipid-like self-assembling peptides," Acc. Chem. Res., vol. 45, pp. 2142-2150, Jun. 2012.

[20] Z. Luo and S. Zhang, "Designer nanomaterials using chiral self-assembling peptide systems and their emerging benefit for society," Chem. Soc. Rev., vol. 41, pp. 4736-4754, May 2012.

[21] D. M. Ryan and B. L. Nilsson, "Self-assembled amino acids and dipeptides as noncovalent hydrogels for tissue engineering," Polym. Chem., vol. 3, pp. 18-33, Oct. 2012.

[22] L. M. Salonen, M. Ellermann, and F. Diederich, "Aromatic rings in chemical and biological recognition: energetics and structures," Angew. Chem. Int. Ed., vol. 50, pp. 4808-4842, May 2011.

[23] K. Reichenbächer, H. I. Süss, and J. Hulliger "Fluorine in crystal engineering - the little atom that could," Chem. Soc. Rev., vol. 34, pp. 22-30, Dec. 2005.

[24] N. Boden, P. P. Davis, C. H. Stam, and G. A. Wesselink, "Solid hexafluorobenzene," Mol. Phys., vol. 25, pp. 81-86, Aug. 1973.

[25] J. H. Williams, J. K. Cockcroft, and A. N. Fitch, "Structure of the lowest temperature phase of the solid benzene-hexafluorobenzene adduct," Angew. Chem., Int. Ed., vol. 31, pp. 1655-1657, Dec. 1992.

[26] J. M. Steed, T. A. Dixon, and W. Klemperer, "Molecular beam studies of benzene dimer, hexafluorobenzene dimer, and benzene-hexafluorobenzene," J. Chem. Phys., vol. 70, pp. 4940-4944, Jul. 1979.

[27] E. Bartsch, H. Bertagnolli, and P. Chieux, Ber. Bunsen-Ges. Phys. Chem., vol. 90, pp. 34-46, 1986.

[28] S.-M. Hsu, Y.-C. Lin, J.-W. Chang, Y.-H .Liu, and H.-C. Lin, "Intramolecular interactions of a phenyl/perfluorophenyl pair in the formation of supramolecular nanofibers and hydrogels," Angew. Chem. Int. Ed., vol. 53, pp. 1921-1927, Feb. 2014

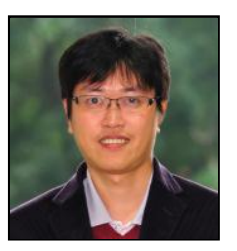

Hsin-Chieh Lin was born in Taipei, Taiwan and received his Ph.D. degree from the National Taiwan University in 2006. He is currently serving as an assistant professor at the Department of Materials Science and Engineering, National Chiao Tung University, Taiwan. His research interests are the development and implementation of tissue repair and regeneration materials, molecular drug delivery systems, cancer therapy, biomedical diagnostics, and biomimetics. The research goal of Lin's group is to improve human health and the quality of life 